\title{
Non-LTE inversion of chromospheric Ca cloud-like features
}

\author{
K. Tziotziou ${ }^{1}$, P. Heinzel ${ }^{2}$, P. Mein ${ }^{1}$, and N. Mein ${ }^{1}$ \\ 1 Observatoire de Paris, Section de Meudon, DASOP, 92195 Meudon Principal Cedex, France \\ 2 Astronomical Institute, Academy of Sciences of the Czech Republic, 25165 Ondřejov, Czech Republic \\ Received 22 June 2000 / Accepted 14 November 2000
}

\begin{abstract}
A chromospheric cloud-like feature observed in the 8542 A Ca II line is studied by a two step inversion procedure which provides estimates of its temperature, electronic density, microturbulence, geometrical thickness and bulk velocity. The first step involves the computation of a large grid of models by a multi-level non-LTE transfer code which gives the Ca II line depth-dependent mean intensity inside an isolated, isothermal cloud lying above the chromosphere. The second step involves the inversion of the observed profiles with the grid of computed synthetic Ca II profiles. A searching and matching $\chi^{2}$ algorithm is implemented followed by an interpolation algorithm which permits a more accurate determination of the parameters on which the profiles depend. The five grid parameters are reduced to four by defining the emission measure from the geometrical thickness and electronic density. We show that this inversion procedure gives accurate results for some of our inversion parameters when dealing with solar filaments and is complementary to a previous study of the same object in the $\mathrm{H} \alpha$ line. The main advantages, problems and future extension of the inversion approach are also discussed.
\end{abstract}

Key words. line: profiles - radiative transfer - methods: data analysis - Sun: chromosphere - Sun: filaments

\section{Introduction}

The formation of chromospheric lines under conditions of non local thermodynamic equilibrium (non-LTE), where the source function decouples completely from the Planck function, requires a careful and complete analysis of the radiative transfer problem. Solar spectral inversion codes, although often do not account for all the physical processes relevant to the radiative transfer nor for the intrinsic 3D structuring, are nevertheless a powerful diagnostic tool for obtaining information for the solar plasma from observed line spectra.

The non-LTE inversion problem has been addressed in several different ways during the past years. A popular tool have been response functions, introduced by Mein (1971), which measure first-order responses of emerging line profiles to perturbations of the atmospheric conditions. They have been used extensively for LTE inversions. Recently Socas-Navarro et al. (1998) used the response function concept with the fixed departure coefficient (FDC) approximation for the non-LTE inversion of several chromospheric line profiles including the Ca II $8542 \AA$ line.

Mein et al. (1987) proposed the decomposition of observed profiles into their Fourier components. Their idea of linking thermodynamical parameters of theoretical model profiles to the Fourier coefficients and studying the corresponding variations was first applied to observed

Send offprint requests to: K. Tziotziou, e-mail: Kostas.Tziotziou@obspm.fr
Ca II 3968 Å line spectra. Briand et al. (1996) further extended the method by using a grid of chromosphere models described by two parameters, namely the temperature at the bottom and the top of the atmosphere, for the non-LTE inversion of Ca II $8542 \AA$ line spectra.

Synthetic profile calculations with multilevel non-LTE transfer codes have been based either on the linearization or on the preconditioning approach. The first approach linearizes all the necessary multilevel transfer equations by neglecting second and higher order terms in the perturbed equations. The multilevel transfer code MULTI developed by Carlsson (1986) is based on this approach. The preconditioning approach linearizes the original non-linear equations by choosing some quantities from a previous iteration and using an approximate relationship for the radiation field. This approach is used by the one-dimensional multilevel transfer code MALI by Heinzel (1995) and has been extended to two-dimensional multilevel radiative transfer by Paletou (1995). Recently Socas-Navarro \& Trujillo Bueno (1997) made a comparison of the two aforementioned approaches and showed that when the same information is taken into account they are essentially the same.

The MALI approach has been recently used by Molowny-Horas et al. (1999) for the non-LTE inversion of a chromospheric $\mathrm{H} \alpha$ cloud-like filament. In cloud models the emerging chromospheric line profiles consist of the background line profiles, partially absorbed by the cloud, and the radiation emitted by the cloud itself. Although the simple cloud model proposed by Beckers (1964) has been 
criticized for failing to explain $\mathrm{H} \alpha$ contrast profiles of chromospheric fine structures (Cram 1975), improved cloud models have been successful for the study of several chromospheric features like filaments (Molowny-Horas et al. 1999), arch filaments (P. Mein et al. 1996; Mein et al. 2000) and mottles (Tsiropoula et al. 1993).

In this paper we are interested in the non-LTE formation of the Ca II $8542 \AA$ line in chromospheric cloud-like features. Hereafter, unless specified otherwise, when referring to the Ca II line we mean the $8542 \AA$ Ca II line. We use the MALI approach to construct a large grid of models which are afterwards compared with observations. We apply the non-LTE inversion procedure to the filament that Molowny-Horas et al. (1999) studied in $\mathrm{H} \alpha$, thus obtaining valuable information about the inversion in different spectral lines.

The outline of the paper is as follows. After this introduction we give a short description of the non-LTE code followed by a description of the grid and the synthetic profile calculations. The inversion procedure is then presented and applied to observed data and we conclude with a discussion of the results.

\section{Ca II MALI code synthetic profile calculations}

For synthetic profile calculations of the Ca II $8542 \AA$ A line we use the Multilevel Accelerated Lambda Iteration (MALI) technique of Rybicki \& Hummer $(1991,1992)$ as it was implemented by Heinzel (1995) for the study of isolated atmospheric structures. A thorough description of the code and its boundary conditions is given in N. Mein et al. (1996), Heinzel et al. (1999) and Molowny-Horas et al. (1999), the application to Ca II is worked out in Mein et al. (2000).

We represent the cloud-like filament by an isothermal horizontal 1D slab (see Fig. 1 in Heinzel et al. 1999), which lies above the solar surface and moves as a whole with a bulk velocity. The input parameters for the synthetic profile calculations are temperature, electron density, microturbulent velocity, macroscopic bulk velocity, geometrical thickness, height above the solar surface and the incident radiation.

We initially solve the non-LTE problem for hydrogen in the same way as Molowny-Horas et al. (1999), in order to compute the radiation field in Lyman lines and the continuum. The computed depth-dependent mean resonance line and continuum intensities are used for the evaluation of the CaII photoionization rates. The non-LTE MALI computation of the Ca II follows, according to Mein et al. (2000).

We consider a five level plus continuum Ca II model (Ca I is much less populated and can be ignored). We refer the reader to Shine \& Linsky (1974) for a complete summary of the Ca II atomic level structure, transition rates, level broadening parameters and photoionization rates used in the MALI code. For the photoionization rate computation we assume that the continua are optically thin (this is also checked numerically afterwards).
As a consequence the rates are defined by the fixed radiation field inside the cloud, as described in Mein et al. (2000). The incident solar radiation field is a compilation of various observations. Partial-redistribution (PRD) effects on atomic level populations are approximated by restricting the resonance-lines frequency range to three Doppler widths and by using the complete redistribution (CRD). Infrared lines are computed with CRD.

The CaII $8542 \AA$ infrared line results from the transition between the fifth energy level $4 \mathrm{p}^{2} P_{3 / 2}$ and the third energy level $3 \mathrm{~d}^{2} D_{5 / 2}$ (in the notation of Shine \& Linsky 1974). The computed non-LTE level populations as a function of the line-center optical depth are used to evaluate the Ca II line source function given by

$S_{\lambda}=\frac{2 h c^{2}}{\lambda^{5}} \cdot \frac{1}{\frac{g_{5} N_{3}}{g_{3} N_{5}}-1}$

where $g_{3}, g_{5}$ are the statistical weights of the respective atomic levels, $N_{3}$ and $N_{5}$ the number densities of Ca II atoms in the third and fifth level, $c$ the speed of light and $h$ Planck's constant. The formal solution of the radiation transfer equation along the line of sight can then be expressed as follows (Molowny-Horas et al. 1999)

$$
\begin{aligned}
I_{\lambda}= & I_{\mathrm{tab}, \lambda} \cdot \mathrm{e}^{-\frac{\tau_{\lambda \max }}{\mu}}+ \\
& \int_{0}^{\tau_{\lambda \max }} S_{\lambda}\left(\tau_{\lambda}\right) \cdot \mathrm{e}^{-\frac{\tau_{\lambda}}{\mu}} \cdot \mathrm{d} \tau_{\lambda} / \mu
\end{aligned}
$$

where $\mu=\cos \theta, \tau_{\lambda}$ is the optical depth and $\tau_{\lambda \max }$ is the optical thickness of the cloud. The background intensity profile $I_{\mathrm{tab}, \lambda}$ has been taken from quiet Sun disk observations (unpublished observations by Gorshkov A. B., Heinzel P. and Vial J.-C.). The optical depth $\tau_{\lambda}$, since the line has a small optical thickness, is expressed by a Doppler-shifted Gaussian profile

$\tau_{\lambda}=\tau_{0} \cdot \mathrm{e}^{-\left(\frac{\lambda-\lambda_{0}-\lambda_{\mathrm{D}}}{W}\right)^{2}}$

where $\tau_{0}$ is the optical depth at line center, $W$ is the line Doppler width

$W=\frac{\lambda_{0}}{c} \cdot \sqrt{\frac{2 k T}{m}+\xi_{\mathrm{t}}^{2}}$,

$\lambda_{0}$ is the CaII line center wavelength $(8542 \AA), \lambda_{\mathrm{D}}$ the Doppler shift of the line due to the line of sight motion of the cloud, $m$ the calcium atom rest mass and $\xi_{\mathrm{t}}$ the microturbulent velocity. For an optically thick cloud in Ca II the shift of the absorption profile would directly give the line of sight velocity but since the cloud is mostly optically thin, as our results indicate, a detailed non-LTE solution is required to determine the actual velocity. Note that $\lambda_{\mathrm{D}}$ is evaluated with the component $V_{\|}$of the true velocity vector as projected into the line of sight. $V_{\|}$can significantly differ from the radial velocity $V$ used as a grid parameter. However, in the example used in the present paper, the filament was observed at disc center $(\mu=1)$ and thus $V$ and $V_{\|}$have the same absolute value. 

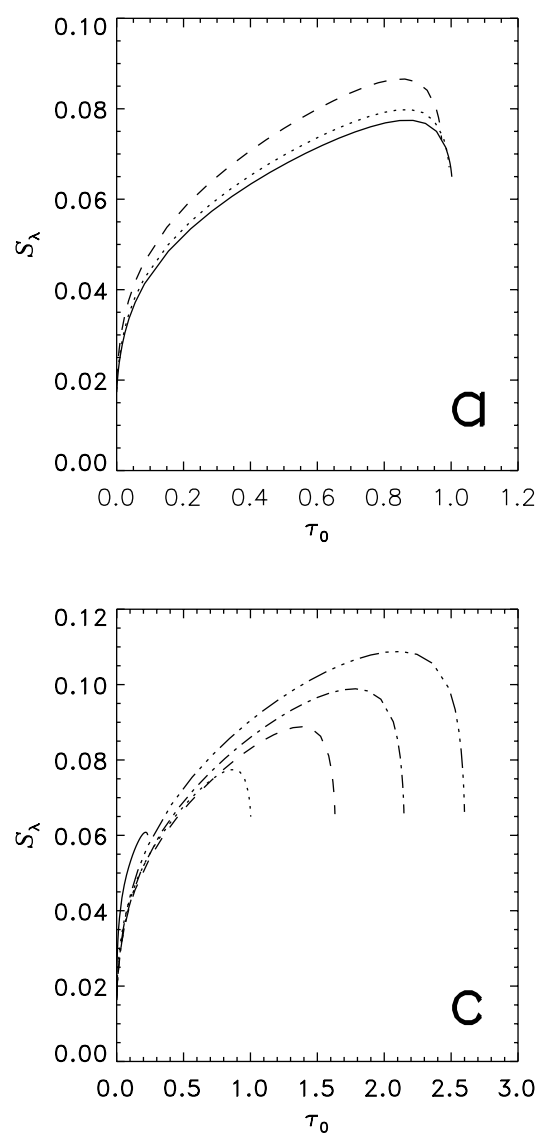

Table 1. Parameters used for the calculation of the grid. The total number of computed MALI models is $3 \times 9 \times 11 \times 5$ $\times 7=10395$. Since the source function is symmetric to negative and positive velocities only the three positive points are calculated for parameter $V$

\begin{tabular}{lccc}
\hline Parameter & Range & Step & Points \\
\hline$V\left(\mathrm{~km} \mathrm{~s}^{-1}\right)$ & $-5-5$ & 2.5 & 3 \\
$N_{\mathrm{e}}\left(\mathrm{cm}^{-3}\right)$ & $210^{10}-110^{11}$ & $110^{10}$ & 9 \\
$T(\mathrm{~K})$ & $6000-11000$ & 500 & 11 \\
$Z(\mathrm{~km})$ & $1000-5000$ & 1000 & 5 \\
$\xi_{\mathrm{t}}\left(\mathrm{km} \mathrm{s}^{-1}\right)$ & $3-9$ & 1 & 7 \\
Height $(\mathrm{km})$ & 20000 & - & 1 \\
$N_{\mathrm{H}}$ & 0.1 & - & 1 \\
$N_{\mathrm{H}}$ & $0^{\circ}$ & - & 1 \\
Ezimuth angle & $0^{\circ}$ & - & 1 \\
\hline
\end{tabular}

\section{Description of the model calculation procedure}

\subsection{The grid of models}

For the Ca II MALI synthetic profile calculations we use cloud velocity $V$, kinetic temperature $T$, electronic density $N_{\mathrm{e}}$, microturbulent velocity $\xi_{\mathrm{t}}$ and geometrical thickness $Z$ of the cloud as input parameters to the code. For the grid calculations, $V$ corresponds to a radial (vertical) component of the true velocity vector. This affects the value of the source function via the Doppler shift of the incident radiation. The range of these parameters, presented in Table 1, gives us a grid of 10395 MALI models. Due to the axial symmetry of the incident radiation, atomic populations of models with the same $T, Z, N_{\mathrm{e}}, \xi_{\mathrm{t}}$ and the same absolute value of $V$ are identical. As a consequence the dependence of all grid model calculations on the sign of $V$ results from the optical depth $\tau_{\lambda}$ in Eq. (3).

In Fig. 1 we show examples of the dependence of the Ca II source function as a function of the line center optical depth on velocity (a), temperature (b) and electronic density (c). The shape of the source function is typical for cloud-like structures seen against the solar disc. For the low velocity values of our calculations, the dependence of the level populations and therefore $S_{\lambda}$ on velocity is not strong and the optical thickness (maximum line center optical depth $\tau_{0}$ ) is practically unchanged. However, as temperature increases the optical thickness of the cloud decreases considerably while for increasing electronic density the optical thickness increases.

To accelerate the MALI computations we use results obtained from the nearest previous model computations as new input estimates for the iteration procedure which is stopped when all populations at all depths reach a relative error of $10^{-3}$. A typical model (hydrogen + Ca II calculations) requires 40 to $60 \mathrm{~s}$ of $\mathrm{CPU}$ time on an Alpha-type workstation running at $400 \mathrm{MHz}$. 


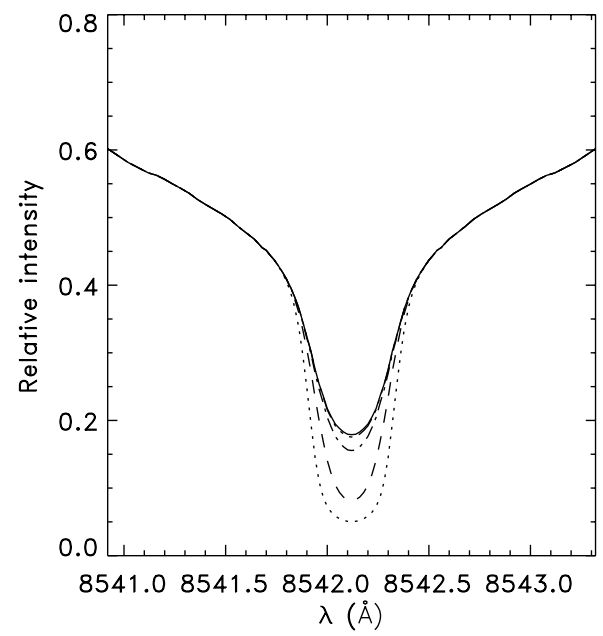

Fig. 2. The Ca II line profile in relative units, for $V=0 \mathrm{~km} \mathrm{~s}^{-1}$, $\xi_{\mathrm{t}}=4 \mathrm{~km} \mathrm{~s}^{-1}, Z=3000 \mathrm{~km}$ and $N_{\mathrm{e}}=310^{10} \mathrm{~cm}^{-3}$. The solid profile corresponds to the CaII chromospheric background while the dotted line corresponds to $T=6000 \mathrm{~K}$, dashed to $T=7500 \mathrm{~K}$, dotted-dashed to $T=9000 \mathrm{~K}$ and three-dotted-dashed to $T=10500 \mathrm{~K}$

\subsection{Analysis of the profiles and their dependence on the grid parameters}

Although the dependence of the CaII profiles on the five input parameters $N_{\mathrm{e}}, T, \xi_{\mathrm{t}}, V$ and $Z$ is not straightforward, a study of the Ca II synthetic profiles for the case of a static $V=0 \mathrm{~km} \mathrm{~s}^{-1}$ cloud shows that:

1. An increase in temperature $T$ gives brighter Ca II intensities near the center of the line (see Fig. 2). There is an increase of the rate of collisions with temperature but since the optical thickness of the cloud decreases with increasing temperature, as Fig. 1b shows, the increase of the line center intensity is mostly due to the lower line center opacity. As a consequence we see more background radiation just like in the case of increasing microturbulence described below. For high enough temperature $T$ and electronic density $N_{\mathrm{e}}$, the line core could go into emission relative to the background but this is never the case for any of our grid model profiles (see also Fig. 4). Since calcium is a much heavier ion than hydrogen the effect of temperature on $W$ through Eq. (4) in the wings of the line is not very strong, as our results also indicate;

2. A similar effect is manifested, as Fig. 3 shows, in the behaviour of the CaII line center intensity as the microturbulent velocity $\xi_{\mathrm{t}}$ increases. An increase of microturbulence causes a considerable broadening of the profile and thus a decrease of the line center opacity. As a consequence, we see more background radiation at the core of the profile. In the wings, contrary to the core, the microturbulent broadening leads to a higher opacity and thus we see less background radiation;

3. The line intensity strongly depends on the electron density $N_{\mathrm{e}}$. As $N_{\mathrm{e}}$ increases, for low temperatures the CaII line center intensity goes through a minimum

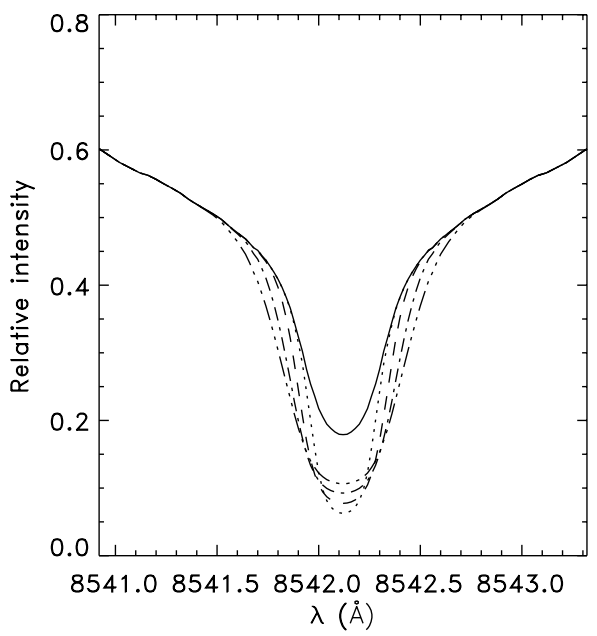

Fig. 3. The Ca II line profile in relative units, for $V=0 \mathrm{~km} \mathrm{~s}^{-1}$, $T=7500 \mathrm{~K}, Z=3000 \mathrm{~km}$ and $N_{\mathrm{e}}=610^{10} \mathrm{~cm}^{-3}$. The solid profile corresponds to the Ca II chromospheric background while the dotted line corresponds to $\xi_{\mathrm{t}}=3 \mathrm{~km} \mathrm{~s}^{-1}$, dashed to $\xi_{\mathrm{t}}=5 \mathrm{~km} \mathrm{~s}^{-1}$, dotted-dashed to $\xi_{\mathrm{t}}=7 \mathrm{~km} \mathrm{~s}^{-1}$, and threedotted-dashed to $\xi_{\mathrm{t}}=9 \mathrm{~km} \mathrm{~s}^{-1}$

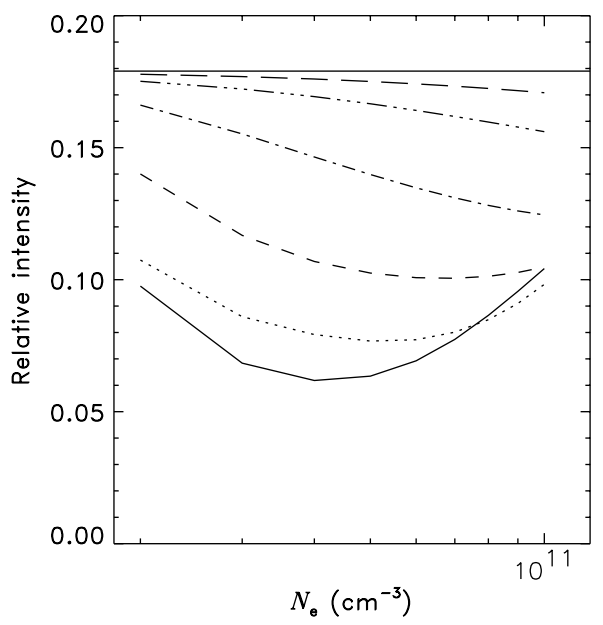

Fig. 4. Intensity of the Ca II line center in relative units as a function of electronic density $N_{\mathrm{e}}$ for the static $V=0 \mathrm{~km} \mathrm{~s}^{-1}$ case. $Z=5000 \mathrm{~km}$ and $\xi_{\mathrm{t}}=9 \mathrm{~km} \mathrm{~s}^{-1}$. The solid curve corresponds to $T=6000 \mathrm{~K}$, dotted to $T=6500 \mathrm{~K}$, dashed to $T=7500 \mathrm{~K}$, dotted-dashed to $T=8500 \mathrm{~K}$, three-dotteddashed to $T=9500 \mathrm{~K}$ and long-dashed to $T=10500 \mathrm{~K}$. The horizontal solid line corresponds to the CaII chromospheric background line center intensity

before increasing again (see Fig. 4) while for high temperatures the line center intensity is monotonically decreasing within the range of used densities. It seems that there is a shift of the minimum seen in the low temperature intensity curves of Fig. 4 towards higher values of $N_{\mathrm{e}}$, beyond our density grid, as temperature increases. A similar behaviour has been observed by Giovanelli (1967) for the $K$ line central intensities of chromospheric model structures.

For low temperatures an increase in density leads initially to an increase of the optical thickness of the cloud and thus absorption until $N_{\mathrm{e}}$ reaches a high 


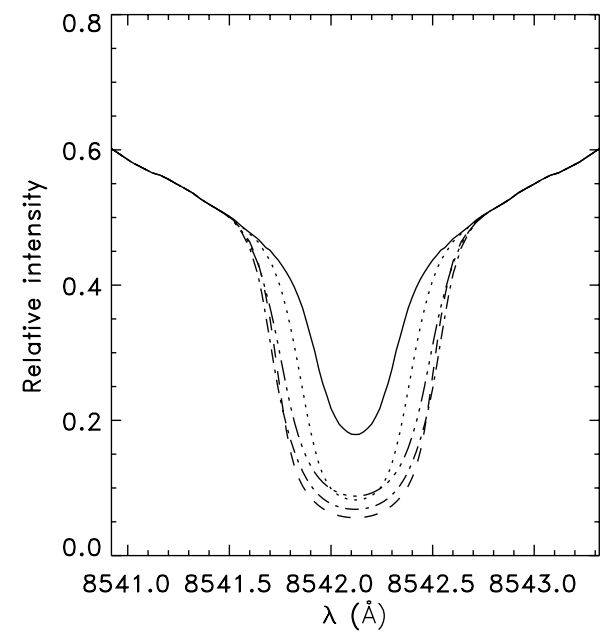

Fig. 5. The Ca II line profile in relative units, for $V=0 \mathrm{~km} \mathrm{~s}^{-1}$, $\xi_{\mathrm{t}}=7 \mathrm{~km} \mathrm{~s}^{-1}, T=6000 \mathrm{~K}$ and $Z=5000 \mathrm{~km}$. The solid profile corresponds to the CaII chromospheric background while the dotted line corresponds to $N_{\mathrm{e}}=210^{10} \mathrm{~cm}^{-3}$, dashed to $N_{\mathrm{e}}=$ $410^{10} \mathrm{~cm}^{-3}$, dotted-dashed to $N_{\mathrm{e}}=610^{10} \mathrm{~cm}^{-3}$ and threedotted-dashed to $N_{\mathrm{e}}=810^{10} \mathrm{~cm}^{-3}$

enough value for collisions to dominate and thus give rise to emission. However, as temperature increases Ca II starts ionizing to Ca III. As a consequence for high temperatures larger electronic densities $N_{\mathrm{e}}$ are needed in order for Ca II to start emitting. In Fig. 5 we show how the above behaviour affects the whole line profile of CaII.

For the range of values used in our calculations there is no contrast reversal (relative to the background chromosphere) for the line center wavelength. However, contrast reversal occurs in certain cases for wavelengths at the wings of the profile, about $\pm 1 \AA$ from the line center. The existence of an intensity minimum in Fig. 4 for low temperatures introduces an ambiguity since an observed line center intensity can be sometimes matched by two different $N_{\mathrm{e}}$ values. However, this is not true for the wings of the profile and thus the use of the whole profile for the inversion should finally give a single value for $N_{\mathrm{e}}$;

4. With increasing geometrical thickness $Z$, as Fig. 6 shows, the Ca II line intensities decrease. This is natural since an increase of $Z$ causes an increase in the optical thickness of the cloud making it less transparent to the incident radiation. The saturation towards larger $Z$ is due to the increase of $\tau_{0}$ of the cloud.

It is obvious from the above analysis of the Ca II infrared line profile dependence on the free parameters $V, N_{\mathrm{e}}, T, \xi_{\mathrm{t}}$ and $Z$ that there can occur ambiguities in the inversion for large uncertainties in the observed profiles. Therefore, the number of variables can be reduced to four by introducing the emission measure $Q$, defined as

$Q=\int_{0}^{Z} N_{\mathrm{e}}^{2} \mathrm{~d} \ell=N_{\mathrm{e}}^{2} Z$.

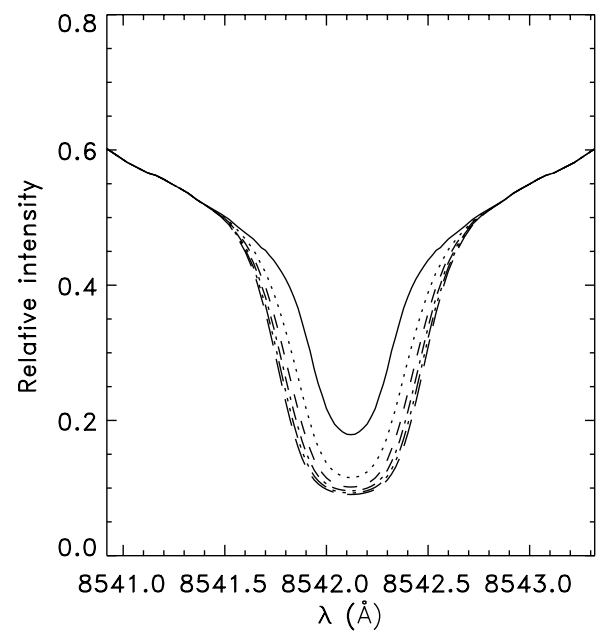

Fig. 6. The Ca II line profile in relative units, for $V=0 \mathrm{~km} \mathrm{~s}^{-1}$, $\xi_{\mathrm{t}}=9 \mathrm{~km} \mathrm{~s}^{-1}, T=7000 \mathrm{~K}$ and $N_{\mathrm{e}}=510^{10} \mathrm{~cm}^{-3}$. The solid profile corresponds to the CaII chromospheric background while the dotted line corresponds to $Z=1000 \mathrm{~km}$, dashed to $Z=2000 \mathrm{~km}$, dotted-dashed to $Z=3000 \mathrm{~km}$, three-dotted-dashed to $Z=4000 \mathrm{~km}$ and long-dashed to $Z=5000 \mathrm{~km}$

The geometrical thickness $Z$ and the electronic density $N_{\mathrm{e}}$ are thus collapsed into a new single parameter (see also Molowny-Horas et al. 1999).

\section{The filament inversion}

\subsection{The inversion procedure}

The comparison of the observed line profile $I_{\mathrm{fil}, \lambda_{i}}$ with each of the grid model profiles $I_{\lambda_{i}}\left(V, T, \xi_{\mathrm{t}}, Q\right)$ is done by a $\chi^{2}$ minimizing function given by

$\chi^{2}=\sum_{i=1}^{N}\left[\frac{I_{\mathrm{fil}, \lambda_{i}}}{I_{\mathrm{bg}, \lambda_{i}}}-\frac{I_{\lambda_{i}}\left(V, T, \xi_{\mathrm{t}}, Q\right)}{I_{\mathrm{tab}, \lambda_{i}}}\right]^{2}$

where $I_{\mathrm{bg}, \lambda_{i}}$ and $I_{\mathrm{tab}, \lambda_{i}}$ are the observed and tabulated background profiles (the latter come from tables, as we have already mentioned, of previous quiet Sun observations), $\lambda_{i}$ refers to the wavelength positions used in the line profile and $N$ is the number of these positions which in our case is equal to five. This $\chi^{2}$ procedure gives the minimum at the resolution of the model grid. A more accurate determination of the parameters is achieved by a parabolic interpolation which takes into account the $\chi^{2}$ values at the nearby grid points.

\subsection{Observational data and the calibration procedure}

The inversion procedure described in the previous section has been applied to a filament observed with the MSDP instrument (Mein 1991) of the German VTT at the Observatorio del Teide on the Canary islands. This observation, made on September 25th, 1996 (also in $\mathrm{Ha}$ line) consists of ten two-dimensional images, recorded simultaneously at 10 wavelengths along the CaII line on 


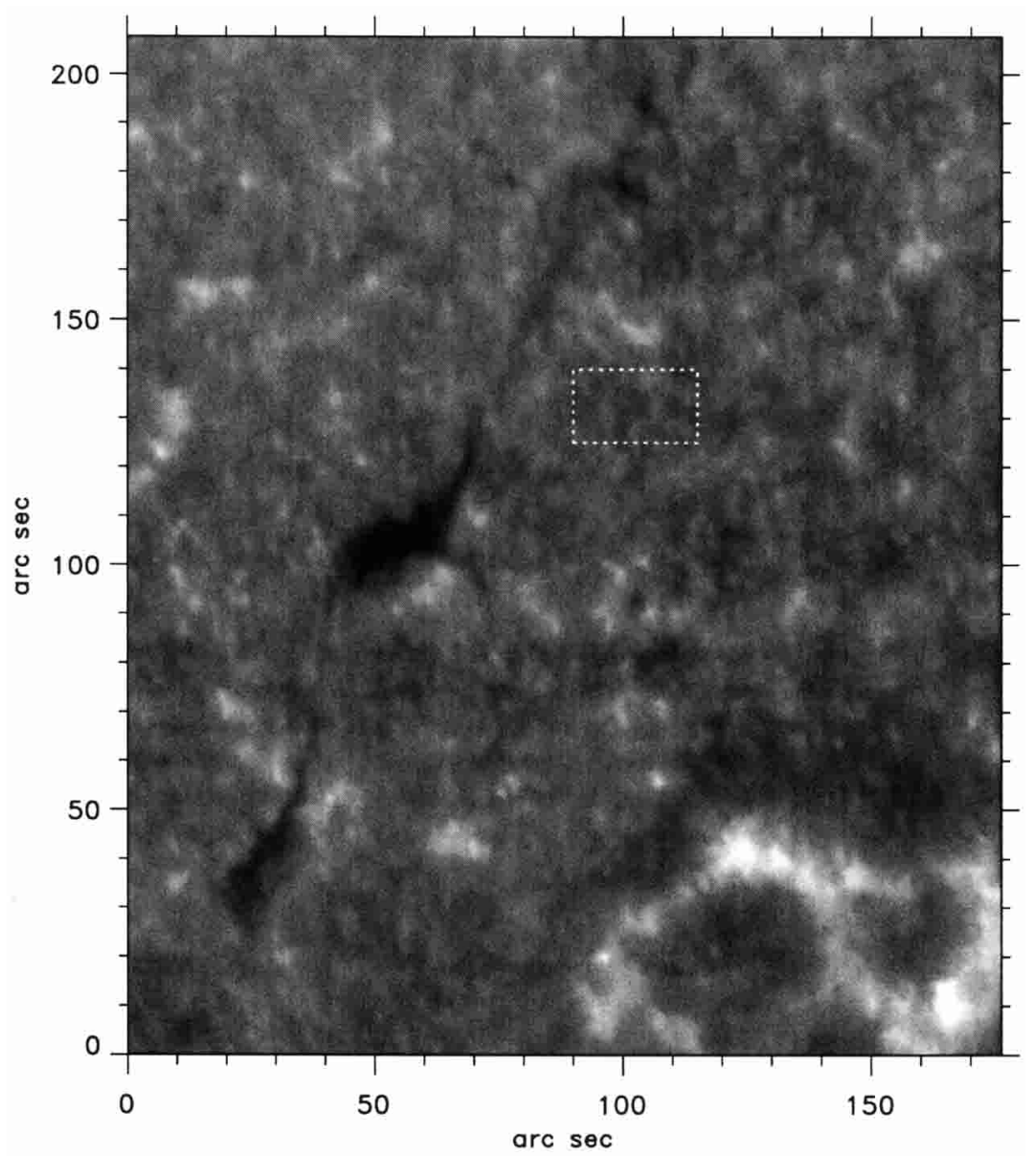

Fig. 7. The MSDP CaII line center image of a solar filament and its surrounding chromospheric regions with a facular region in the lower right part of the figure (bright patch). To compute the background CaII profile we used the region inside the dotted rectangular box

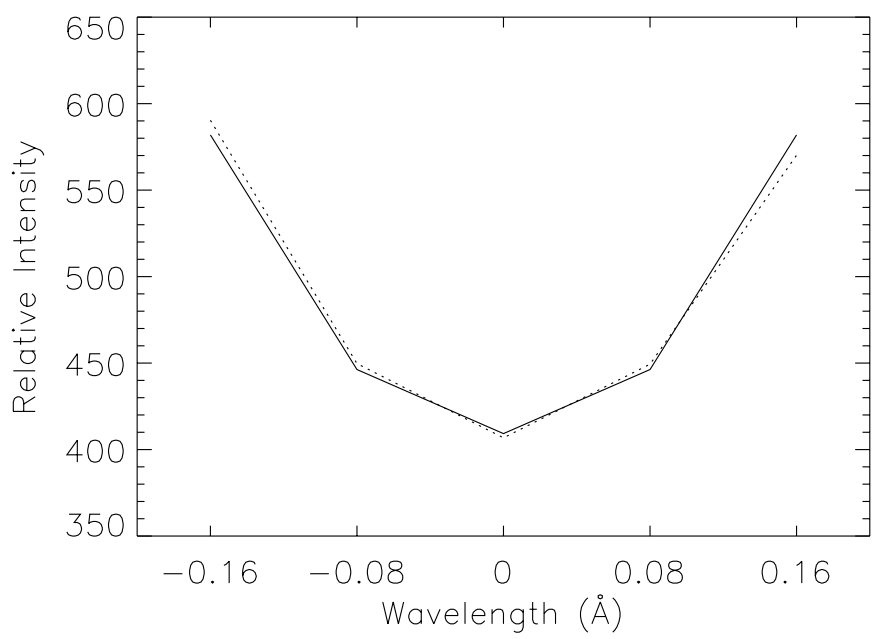

Fig. 8. Relative intensity of the tabulated Ca II background profile (solid line) and of the present MSDP study (dotted line) in arbitrary units. The tabulated Ca II profile has been corrected with the proportionality factor $K$ (see text). All wavelengths are given relative to Ca II line center $(8542 \AA)$

a $1024 \times 1024$ pixel CCD camera. The spatial resolution was 0.44 arcsec, the distance between the channels was about $80 \mathrm{~m} \AA$ and the local bandwidth about $40 \mathrm{~m} \AA$. A large area was scanned in short time intervals and furthermore the CaII line profile was determined at every pixel point of the final image. Only five wavelengths are considered for our study due to a lack of contrast for the far wing wavelengths of the line.

In Fig. 7 we present an $178 \times 210$ arcsec image of the observed filament in the Ca II line center wavelength. The mean Ca II background profile was calculated from pixels inside the dotted rectangular box which is well away from the visible lower right side facular region.

We have simulated on the theoretical profiles the effect of observing with the MSDP instrument by integrating them numerically in intervals of $0.04 \AA$, centered at wavelengths of $-0.16 \AA,-0.08 \AA, 0 \AA,+0.08 \AA$ and $+0.16 \AA$ relative to the Ca II line center at $8542 \AA$. A calibration of the observed and calculated profiles can be performed by comparing the observed and tabulated background profiles $I_{\mathrm{bg}, \lambda_{i}}$ and $I_{\mathrm{tab}, \lambda_{i}}$. We use a proportionality factor

$K=\frac{\sum_{i=1}^{N} I_{\mathrm{bg}, \lambda_{i}} \cdot I_{\mathrm{tab}, \lambda_{i}}}{\sum_{i=1}^{N}\left(I_{\mathrm{tab}, \lambda_{i}}\right)^{2}}$

to calibrate all observed profile intensities with the grid model intensities. Figure 8 compares the observed Ca II background profile with the tabulated background profile used for the grid model calculations. The difference of the observed and the tabulated profile is always less 

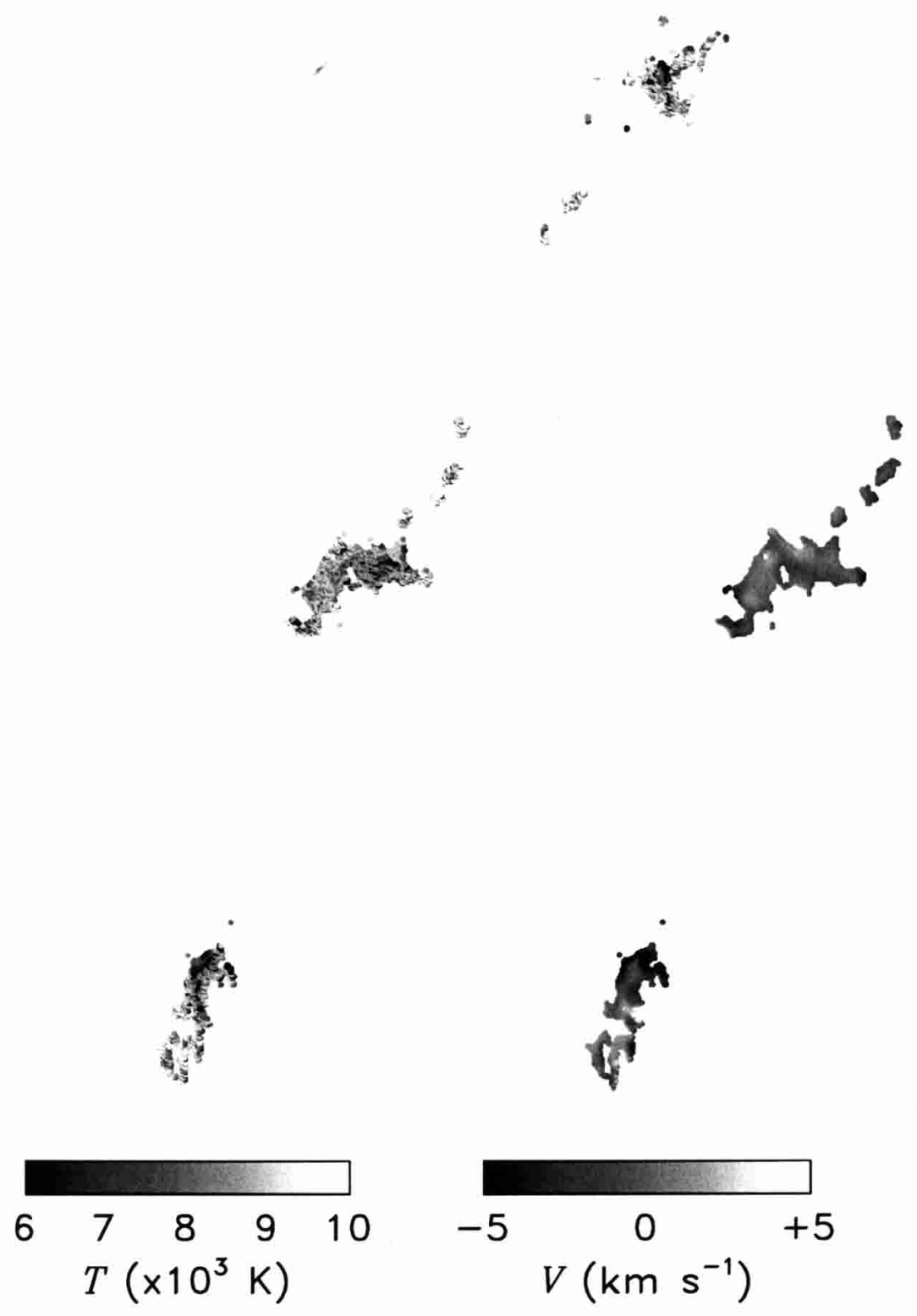

Fig. 9. Maps of the temperature $(T)$ and velocity $(V)$ distribution inside the filament body according to the inversion of filament Ca II profiles. Negative and positive velocities correspond to velocities towards and away from the observer. Only points within the filament that have a relative intensity to the background intensity in CaII of less than 0.95 are considered than $2 \%$ and can be attributed to instrumental or observational reasons (correction for dark current and flat field, stray light, variable seeing, possible inhomogeneities in the used quiet sun region, etc.). The small difference of the two background profiles suggests that we can actually directly use - when possible - the contrast profiles (relative to the background) for the inversion instead of the actual calibrated intensities.

\subsection{Data inversion}

We have inverted a total of 9555 Ca II profiles from the filament region of Fig. 7. In Fig. 9 we show the resulting distributions of temperature and bulk velocity. In Fig. 10 we present the distributions of microturbulent velocity and emission measure inside the filament body. These distributions and all results presented hereafter of the filament inversion include only those filament points that have a maximum relative intensity to the background intensity over the profile lower than 0.95 (a total of 4009 profiles). The implementation of this condition ensures the exclusion of all border points of the filament of Fig. 7, where the filament is extremely optically thin and we may be observing the background.

A first qualitative look at the obtained parameter maps shows that:

- The darker center of the filament seems to have lower temperatures than its borders. This is in agreement 

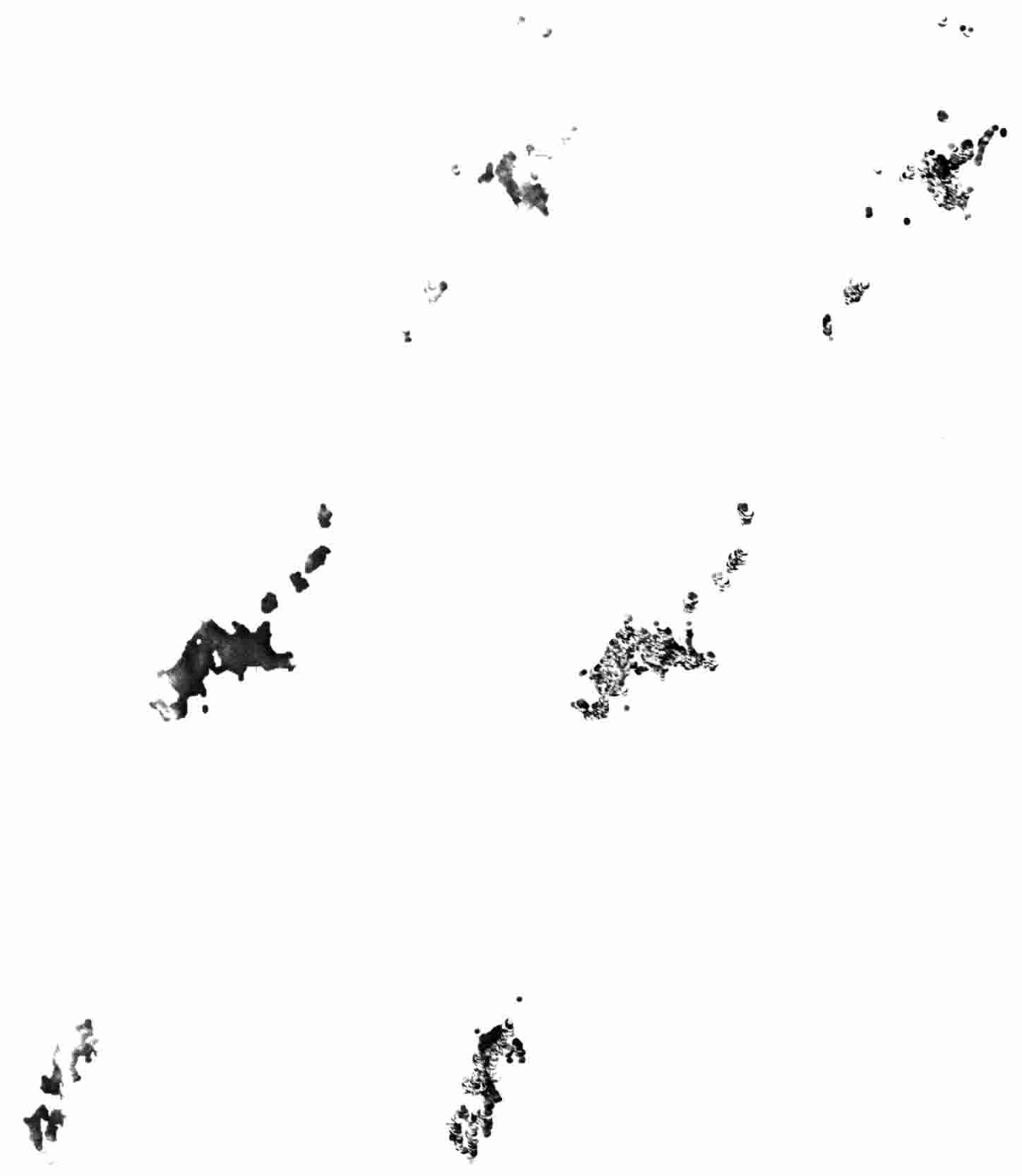
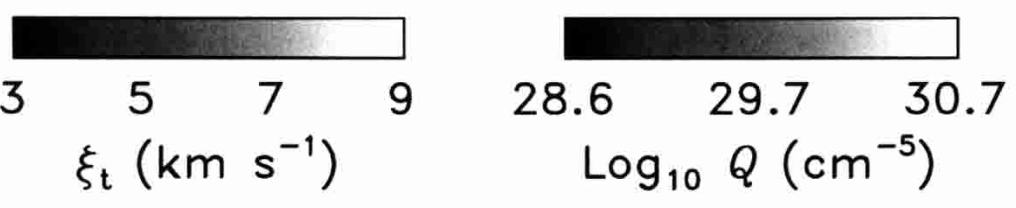

Fig. 10. Maps of the inverted microturbulence $\left(\xi_{\mathrm{t}}\right)$ and emission measure $(Q)$ distribution inside the filament body. Only points within the filament that have a relative intensity to the background intensity in $\mathrm{Ca}$ II of less than 0.95 are considered with the results obtained for $\mathrm{H} \alpha$ by Molowny-Horas et al. (1999);

- We can roughly recognize the same velocity structures seen by Molowny-Horas et al. (1999) for $\mathrm{H} \alpha$;

- Although there is no clear pattern in the distribution of $\xi_{\mathrm{t}}$ within the filament, the filament borders seem to have higher values of microturbulence;

- There is no trend at all in the distribution of emission measure $Q$. This is in contrast with the results of Molowny-Horas et al. (1999) for $\mathrm{H} \alpha$ where $Q$ clearly increases from the border towards the center of the filament. We will further discuss the inversion of $Q$ and its problems in Sect. 4.3.1.
A direct comparison of the inverted parameter values for $\mathrm{Ca}$ II and for $\mathrm{H} \alpha$ at every point in the filament is usually not possible since we are probably not looking at the same parts of it. This is mostly attributed to different formation depths for the $\mathrm{H} \alpha$ and CaII lines within the filament as well as to the different refraction indices of the two lines in Earth's atmosphere. However a statistical analysis of the results for the two lines, shown in Fig. 9, reveals that:

1. The temperature in the Ca II distribution peaks around $8500 \mathrm{~K}$ and then the number of inverted profiles drops dramatically for higher temperatures. This is expected since Ca II is mostly dominant for low temperatures and dramatically drops for higher temperatures where 

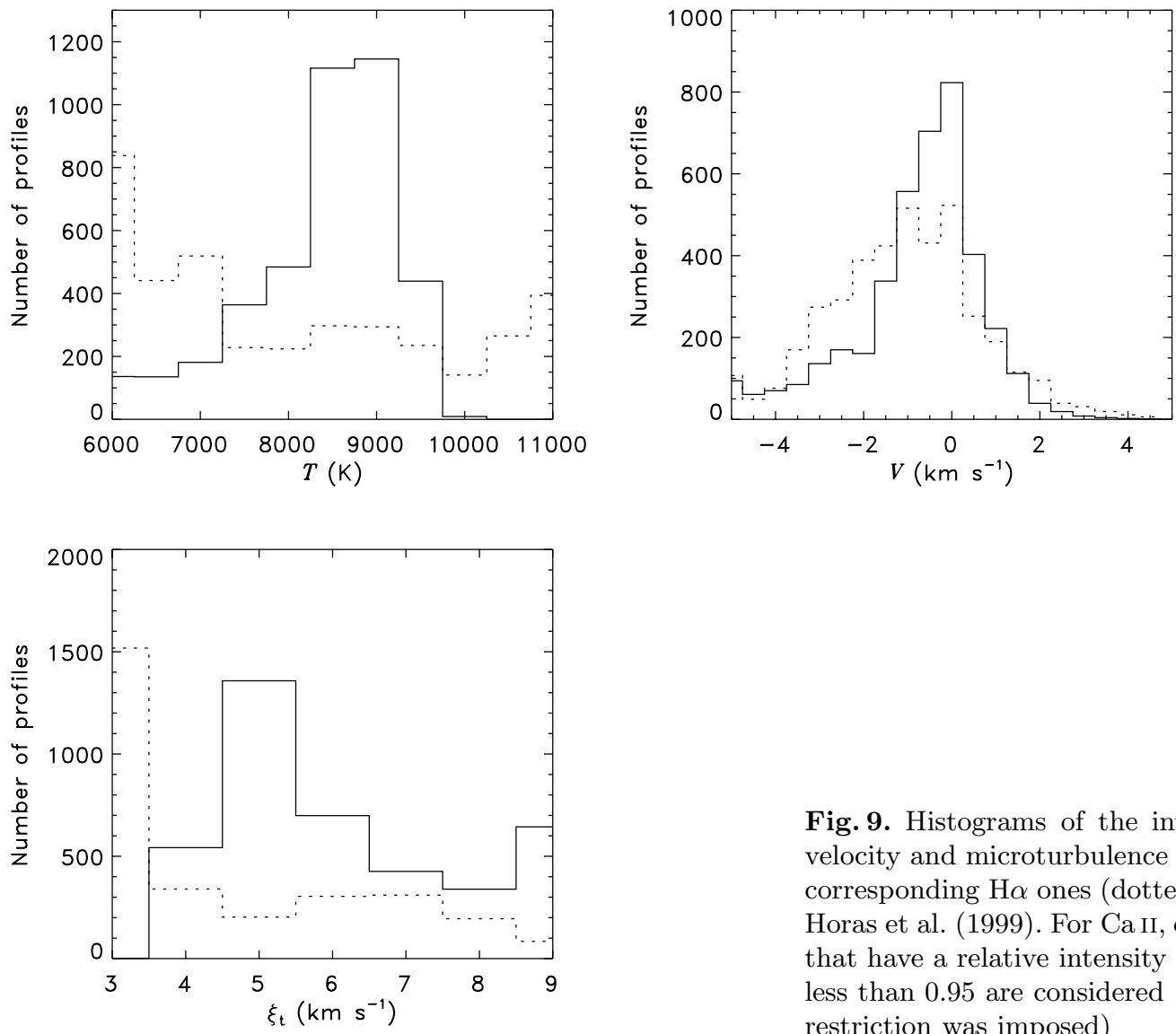

Fig. 9. Histograms of the inverted values of temperature, velocity and microturbulence for Ca II (solid lines) and their corresponding $\mathrm{H} \alpha$ ones (dotted lines) obtained by MolownyHoras et al. (1999). For Ca II, only points within the filament that have a relative intensity to the background intensity of less than 0.95 are considered (for the $\mathrm{H} \alpha$ inversions no such restriction was imposed)

the formation of CaIII is more favorable. Moreover, Fig. 4 shows that within the selected range of parameters the inversion should provide quite accurate values for temperature, since for a specific density there is a clear and strong drop of the CaII intensity as temperature decreases. In the $\mathrm{H} \alpha$ distribution there is no clear equivalent peak in temperature and the filament seems to be on average cooler. Figure 4 of MolownyHoras et al. (1999) shows that the drop of $\mathrm{H} \alpha$ intensity as temperature drops, for a specific density within the same range as our CaII analysis, is not as strong as in the case of Ca II. The different behaviour of the two lines is explained by the fact that the CaII lines are more coupled to the kinetic temperature compared to $\mathrm{H} \alpha$ which is photoionization dominated (Mihalas 1978);

2. A peak is also seen in the microturbulence distribution of CaII around $5 \mathrm{kms}^{-1}$. Since temperature is well defined by the CaII inversion it leads to a better definition of the microturbulence from the profile width, defined by Eq. (4), which depends on temperature and microturbulence. The coupling of temperature and microturbulence is stronger in the case of $\mathrm{H} \alpha$ which is a much lighter element than CaII. As a result the corresponding $\mathrm{H} \alpha$ microturbulence distribution does not peak as there are several couples of values for temperature and microturbulence that correspond to a specific line width;

3. The velocity distribution of Ca II seems to be Gaussian like. The equivalent velocity distribution for $\mathrm{H} \alpha$ shows an excess of blue shifted velocities (filament moving upwards) something clearly seen also in the $\mathrm{H} \alpha$ Doppler velocity maps of the observations. The CaII velocity distribution appears to have slightly more blue shifted velocities as well, but it is not a trend defined as clearly as in the case of $\mathrm{H} \alpha$. However, we should take into account that for the optically thinner CaII, where the optical thickness is on average less than one, velocity structures of the background play an important role for the velocity determination in the filament itself.

\subsubsection{Inversion uniqueness and error analysis}

The uniqueness and precision of the inversion procedure can be checked by exploring the whole parameter space. In Fig. 10 we present for an observed filament profile the 

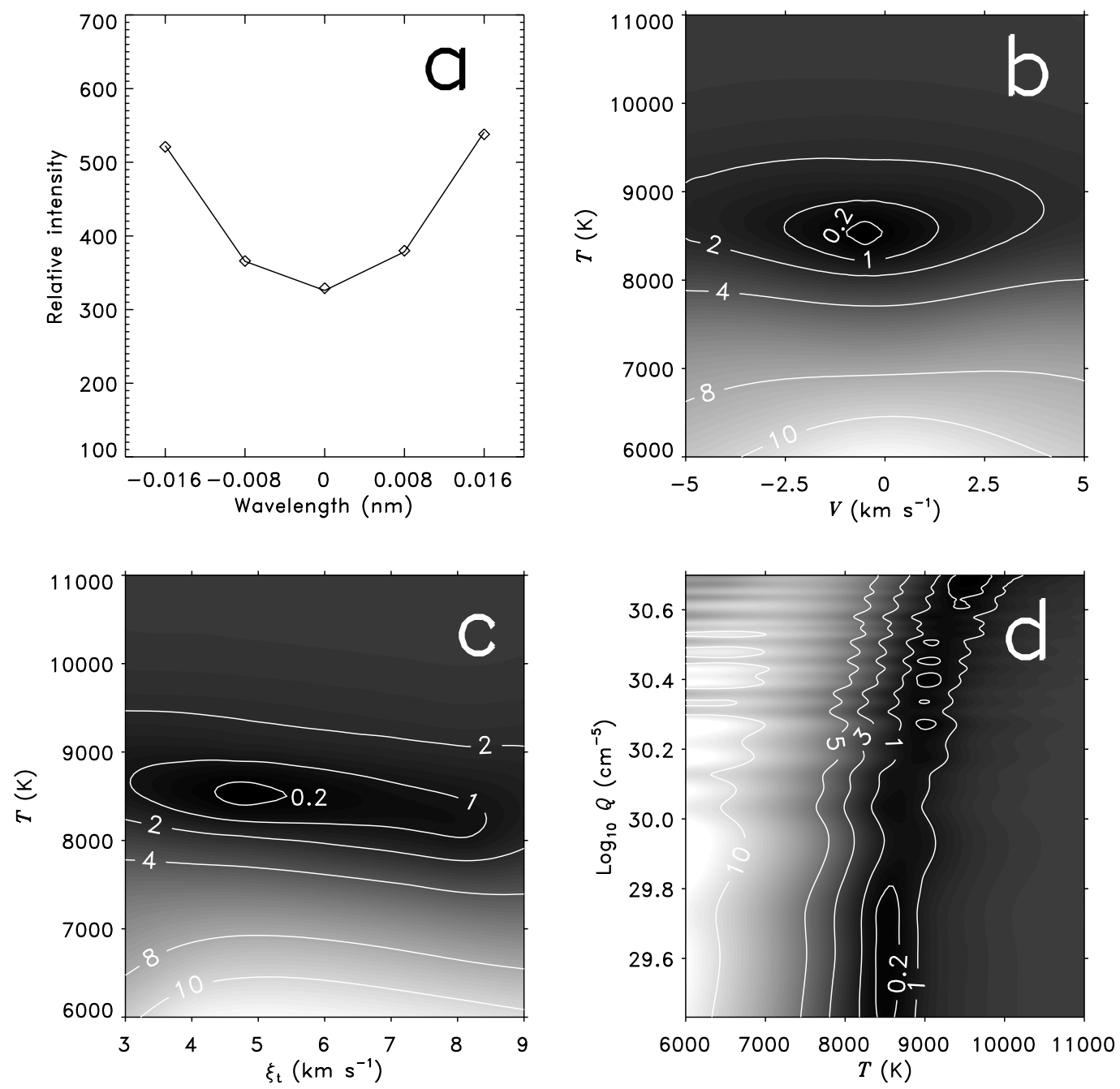

Fig. 10. a) An observed Ca II profile (diamonds) from the filament and the corresponding MALI grid profile (solid line) that minimizes the $\chi$ function of Eq. (8). The inverted parameters values are: $V=0.51 \mathrm{~km} \mathrm{~s}^{-1}, \xi_{\mathrm{t}}=5.29 \mathrm{~km} \mathrm{~s}{ }^{-1}, T=8617 \mathrm{~K}$ and $\log Q=29.53$. $V-T$ cut $\mathbf{b}), \xi_{\mathrm{t}}-T$ cut $\left.\mathbf{c}\right)$ and $T-Q$ cut d) through the $\chi$ distribution for the profile presented in a). Darker tones reveal lower $\chi$ values and the contours denote intensity differences between the observed and the MALI grid profiles normalized in units of $1 \%$ of the nearby CaII continuum

$V-T, \xi_{\mathrm{t}}-T$ and $T-Q$ planes of the 4-dimensional $\chi$ distribution described by

$\chi=\sqrt{\frac{1}{5} \sum_{i=1}^{N}\left[\frac{\frac{I_{\mathrm{fil}, \lambda_{i}}}{K}-I_{\lambda_{i}}\left(V, T, \xi_{\mathrm{t}}, Q\right)}{\delta_{i}}\right]^{2}}$

where $\delta_{i}$ represent the measurement errors and $K$ is the multiplicative factor calculated in 4.2 . We consider $\delta_{i}$ constant for all $i$ 's and equal to $1 \%$ of the intensity of the nearby Ca II continuum. As we can see in Fig. 10 the temperature is always well defined by the inversion procedure. The inversion procedure gives only one solution within the range of parameters and defines quite accurately the global minimum of the $\chi$ distribution for velocity, temperature and microturbulence. However it fails, as clearly seen in Fig. 4.2, to define the value of emission measure $Q$. As Fig. 10d shows, we have several local minima for $Q$ and as a consequence small fluctuations of temperature result in a wide range of values for $Q$, contrary to the case of $\mathrm{H} \alpha$ where the emission measure is always well defined. As Molowny-Horas et al. (1999) demonstrated there is a clear relationship between the integrated $\mathrm{H} \alpha$ intensity emitted by the slab itself and $Q$ while the corresponding integrated Ca II intensity, presented here in Fig. 11, shows no correlation at all. This is again related to the different nature of both lines. The almost unique correlation found for $\mathrm{H} \alpha$ means that for a given $Q$, the integrated intensity is almost insensitive to kinetic temperature. The reason is that $\mathrm{H} \alpha$ is photoionization dominated (for details see Heinzel et al. 1994). The CaII line is, on the other hand, coupled to the kinetic temperature via collisions. We should point out that all the wrinkles seen in the contours of Fig. 10d (as well as in Fig. 13) for high values of $Q$ are mainly caused by the contour interpolation of a highly irregular grid for large values of emission measure. 


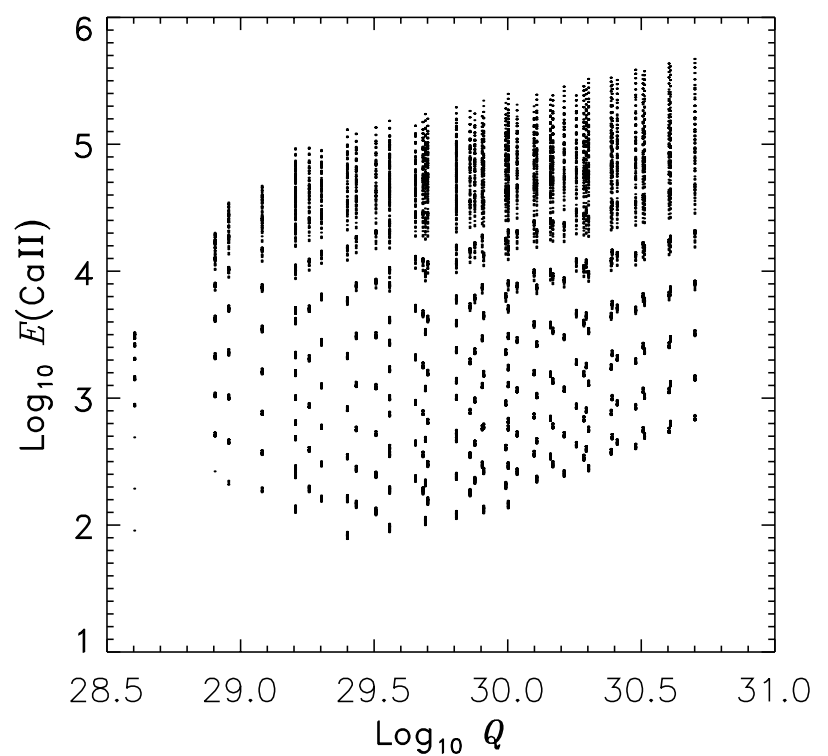

Fig. 11. Integrated intensity $E$ (CaII) emitted by the slab itself, (second term on the right-hand-side of Eq. (2)), vs. $Q$. $E(\mathrm{CaII})$ is given in units of $\mathrm{erg} \mathrm{s}^{-1} \mathrm{~cm}^{-2} \mathrm{sr}^{-1}$ and $Q$ in units of $\mathrm{cm}^{-5}$

A better and even more convincing investigation of the whole parameter space can be done by the calculation for each of our inverted parameters $\left(V, T, \xi_{\mathrm{t}}, Q\right)$ of the standard deviation

$\sigma_{x}=\sqrt{\frac{1}{N-1} \sum_{j=1}^{N}\left(x_{j}-x_{0}\right)^{2}}$

where $x_{0}$ is the global minimum value of our parameter for each inverted filament profile and $x, N$ are respectively the parameter distribution and the corresponding number of solutions that have an intensity difference within a certain percentage of the observed profile intensity. In Fig. 12 we show the standard deviation distributions for our four inversion parameters. Solutions with an intensity which is on average over the whole profile within $2 \%$ of the observed profile intensity are considered. The calculated average standard deviation value is $0.45 \mathrm{~km} \mathrm{~s}^{-1}$ for velocity, $0.61 \mathrm{~km} \mathrm{~s}^{-1}$ for microturbulence, $918 \mathrm{~K}$ for temperature and $1.7510^{30} \mathrm{~cm}^{-5}$ for the emission measure. Even for such a large error the average standard deviation is quite low for velocity, microturbulence and temperature but very high, comparable to the actual range, for emission measure.

\subsubsection{A new inversion parameter for Ca II}

It is quite evident from the results presented so far and their interpretation that the inversion of emission measure for the Ca II line is not possible. However, a parameter that could always be inverted is the maximum line-center optical depth $\tau_{0 \text {,max }}$. This is proportional to the line-center absorption coefficient which depends on the lower level population, microturbulence and temperature. The lower level population is strongly coupled with $N_{\mathrm{e}}$ and $T$. In
Fig. 13 we present $\tau_{0, \max }$ as a function of emission measure $Q$ and temperature $T$ for two different values of microturbulence. As $\xi_{\mathrm{t}}$ increases the values of $\tau_{0, \max }$ decrease. The figure clearly shows that for a given value of $\tau_{0, \max }$ small fluctuations of temperature correspond to a wide range of values of $Q$, another striking proof of the inability of the inversion procedure for $Q$ in the Ca II line.

In Fig. 14 we show the inverted $\tau_{0, \max }$ distribution inside the filament body. The value of $\tau_{0, \max }$ ranges from 0.1 to 1.14 within the filament and it is generally higher in the center of the filament than its borders. This is in absolute agreement with previous observations of filaments.

\section{Discussion}

The inversion strategy for the CaII filament with the use of a grid of models permits a quick and quite accurate search for the best fit to a large amount of observational data. Moreover, it allows an in-depth study of the parameter space and the inter-dependences of parameters. This study can provide useful information concerning the importance of each free parameter to the formation of the CaII line and all the possible physical constraints. The grid of models gives the opportunity to accurately distinguish between local and global minima of the parameter space as it has been clearly demonstrated in 4.3.1. This detailed exploration allows us to deduce that, while the inversion of temperature and microturbulence for Ca II is more accurate than for $\mathrm{H} \alpha$, the inversion of emission measure totally fails. However, we should keep in mind that the range of temperatures for the formation of the CaII line is more restricted than the $\mathrm{H} \alpha$ ones, a trend clearly seen in the results.

The analysis of the results implies that simultaneous observations of dark cloud-like features in both lines could be a useful tool to the understanding of their physical structure. Observations in $\mathrm{H} \alpha$ could give an accurate determination of the electronic density of those features, allowing a more accurate determination of temperature and microturbulence from Ca II observations. This is of course a simplified view since by observing in different lines we are probably attacking physically different structures within the cloud-like features. The formation of hydrogen lines obeys different physical rules than the formation of calcium. One should be careful in applying directly the results obtained for a specific line to another before fully understanding the physical conditions and assumptions under which this could possibly be done.

The filling factor of the filament is another parameter that should be taken into consideration. We could assume that the filament consists of small clouds, each to be treated in the way presented in this paper. For a thin filament, where the individual clumps of matter are far away from each other, we could then assume that each one will only experience the incident background radiation. In this case we would only have to sum up contributions to the 

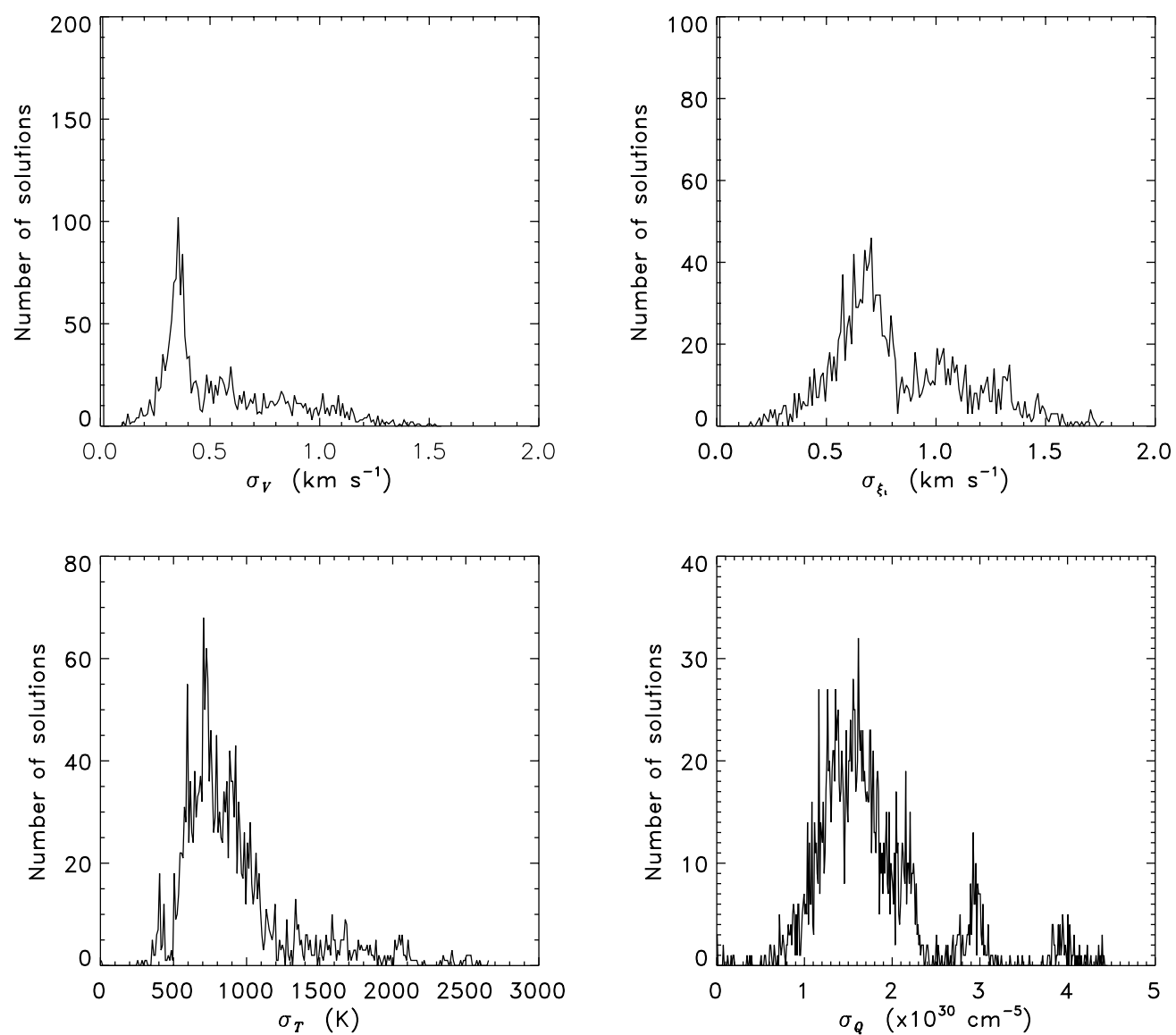

Fig. 12. The standard deviation $\sigma$ distributions for velocity, temperature, microturbulence and emission measure for all grid models with an intensity within $2 \%$ of the observed intensity at each inverted filament point
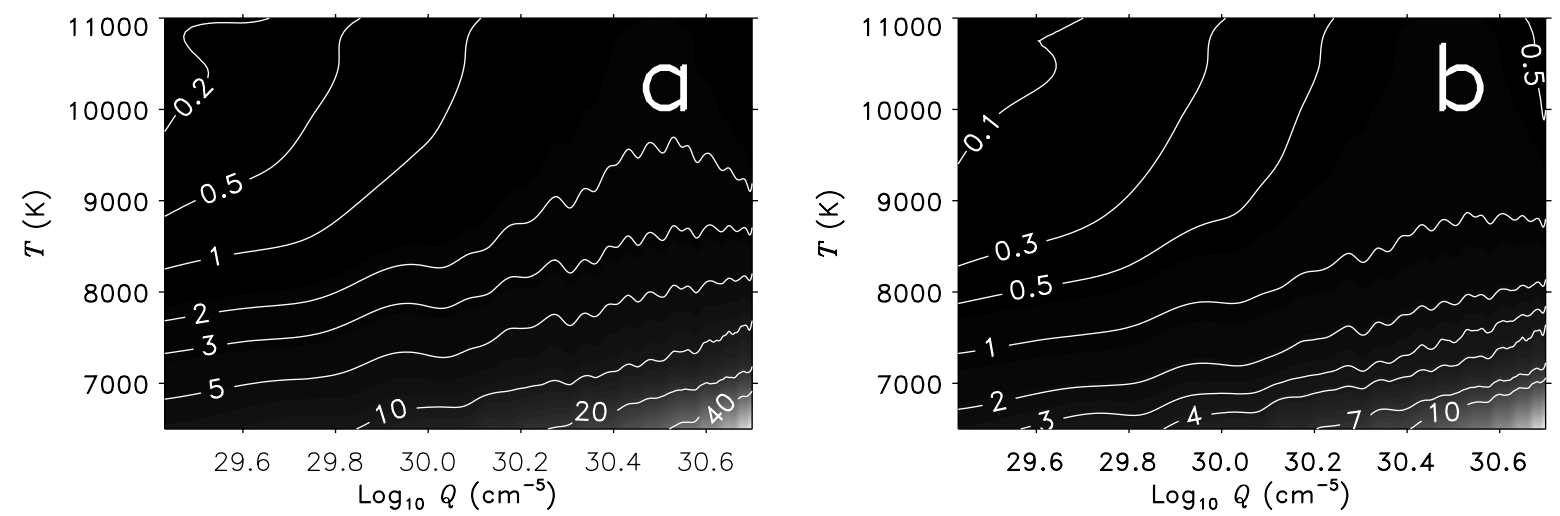

Fig. 13. The maximum line-center optical depth $\tau_{0, \max }$ as a function of emission measure $Q$ and temperature $T$ for microturbulence $\xi_{\mathrm{t}}=3 \mathrm{~km} \mathrm{~s}^{-1}$ a) and $\left.\xi_{\mathrm{t}}=9 \mathrm{~km} \mathrm{~s}^{-1} \mathbf{b}\right)$

line profile from clouds along the line of sight. However, in case of a dense filament each individual cloud would experience the radiation coming from its neighboring ones, thus making the computations extremely complicated. For the purposes of this paper the filling factor was ignored.

Future investigations in Ca II should probably include a grid extended to lower densities. Since cloud-like features are usually optically thin in Ca II, Fig. 13 clearly suggests that we have to allow for densities below $210^{10} \mathrm{~cm}^{-3}$. This figure actually allows the determination of the optimal space of parameters that needs to be investigated if the maximum optical depth is known.

The inversion of velocity in Ca II suggests that a more careful consideration of the background intensity should 

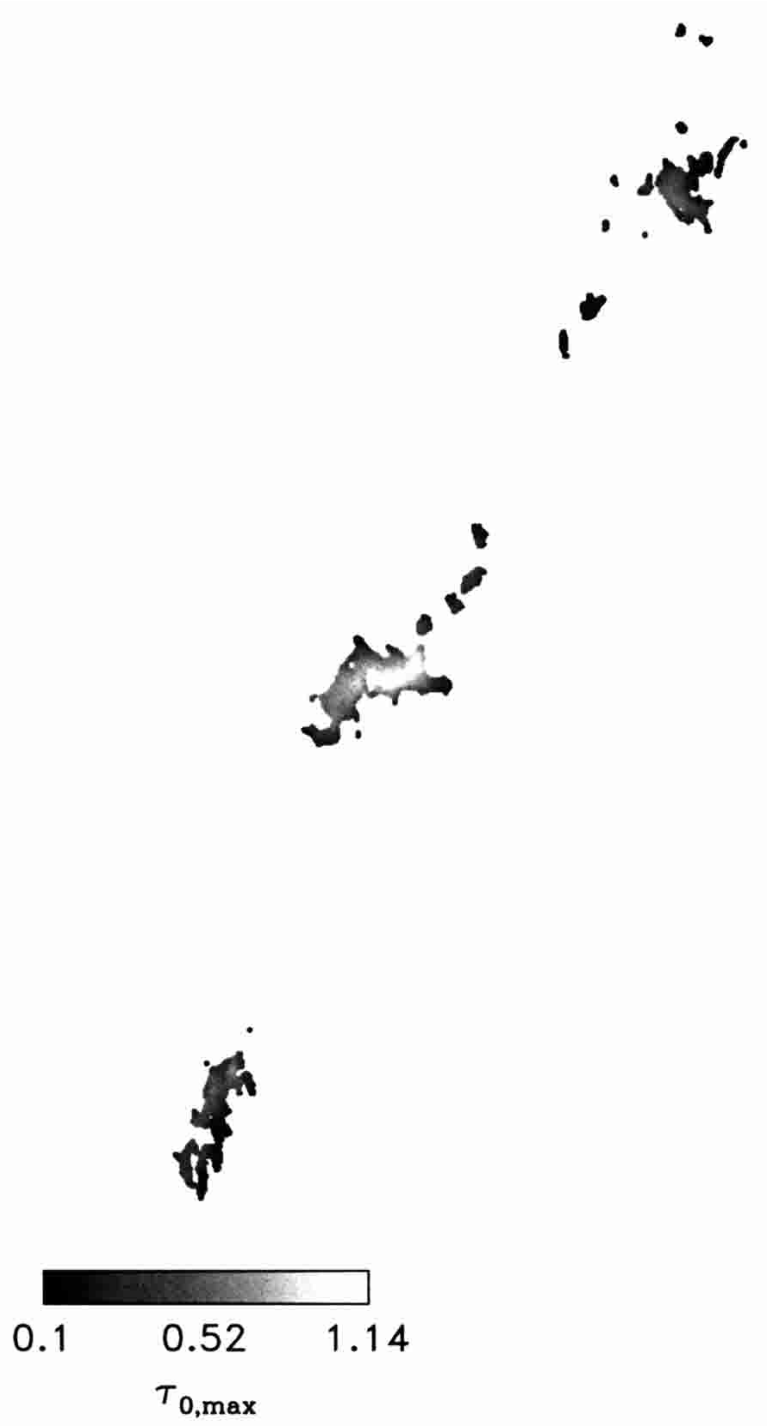

Fig. 14. Map of the maximum line-center optical depth $\tau_{0, \max }$ distribution inside the filament body according to the inversion of filament Ca II profiles

be taken into account. Since the background in CaII is quite structured, as Fig. 7 clearly shows, that influences the CaII profiles from optically thin parts of the filament. It introduces several complicated modifications to the line profile through different illumination conditions and Doppler shifts existing in the incident radiation profiles. The incident radiation, which comes from a large area on the solar surface, is important for the non-LTE boundary conditions in calculating the source function. However, for the transmitted (partially absorbed) radiation of optically thin structures, we need the actual background intensity below the filament for the along the line of sight integration of Eq. (2). A future extension of the inversion code will include this background radiation as a free parameter, something that should definitely lead to a more accurate determination of the actual velocity structure within cloud-like features.
Moreover, an extension of the grid to higher velocities combined with the inclusion of background radiation as an input parameter would enable the investigation of a wide range of chromospheric cloud-like features apart from filaments.

Acknowledgements. MSDP observations were obtained in the framework of the International Observing Time offered by the CCI of the Canarian Observatories and supported by the European Commission through the Access to LargeScale Facility "Activity of the Human Capital and Mobility Program". The Vakuum-Turm-Teleskop (VTT) is operated on the island of Tenerife by the Kiepenheuer-Institut für Sonnenphysik at the Spanish Observatorio del Teide of the Instituto de Astrofísica de Canarias. PH was supported by the CNRS and by the project K1-003-601 of the Academy of Sciences of the Czech Republic. This research is part of the European Solar Magnetometry Network supported by the EC through the TMR programme. The authors are grateful to the referee, Dr. J. Bruls, for his comments and suggestions.

\section{References}

Beckers, J. M. 1964, Ph.D. Thesis, University of Utrecht Briand, C., Mein, P., \& Malherbe, J. M. 1996, in Science with Themis, ed. N. Mein, \& S. Sahal-Bréchot, Observatoire de Paris-Meudon

Carlsson, M. 1986, A Computed Program for Solving Multilevel Non-LTE Radiative Transfer Problems in Moving or Static Atmospheres, Upsala Astronomical Observatory Report, No. 33

Cram, L. E. 1975, Solar Phys., 42, 53

Giovanelli, R. G. 1967, in Solar Phys., ed. J. N. Xanthakis, Interscience, London

Heinzel, P. 1995, A\&A, 299, 563

Heinzel, P., Gouttebroze, P., \& Vial, J.-C. 1994, A\&A, 292, 656

Heinzel, P., Mein, N., \& Mein, P. 1999, A\&A, 346, 322

Mein, N., Mein, P., Heinzel, P., et al. 1996, A\&A, 309, 275

Mein, P. 1971, Solar Phys., 20, 3

Mein, P. 1991, A\&A, 248, 669

Mein, P., Briand, C., Heinzel, P., \& Mein, N. 2000, A\&A, 355, 1146

Mein, P., Démoulin, P., Mein, N., et al. 1996, A\&A, 305, 343

Mein, P., Mein, N., Malherbe, J. M., \& Dame, L. 1987, A\&A, 177,283

Mihalas, D. 1978, Stellar Atmospheres, 2nd edition (W. H. Freeman, San Fransisco)

Molowny-Horas, R., Heinzel, P., Mein, P., \& Mein, N. 1999, A\&A, 345, 618

Paletou, F. 1995, A\&A, 302, 587

Rybicki, G. B., \& Hummer, D. G. 1991, A\&A, 245, 171

Rybicki, G. B., \& Hummer, D. G. 1992, A\&A, 262, 209

Socas-Navarro, H., \& Trujillo Bueno, J. 1997, ApJ, 490, 383

Socas-Navarro, H., Ruiz Cobo, B., \& Trujillo Bueno, J. 1998, ApJ, 507, 470

Shine, R. A., \& Linsky, J. L. 1974, Solar Phys., 39, 49

Tsiropoula, G., Alissandrakis, C. E., \& Schmieder, B. 1993, A\&A, 271, 574 